\section{THU0233 ANTIBODIES AGAINST COMMENSAL STREPTOCOCCAL SPECIFIC PROTEIN (SSP) AND MITOCHONDRIAL IMMUNO- DOMINANT PROTEIN(MIP) IN DIAGNOSIS OF SYSTEMIC LUPUS ERYTHEMATOSUS (SLE) AND OTHER SYSTEMIC AUTOIMMUNE DISEASES}

C. H. Hsu', C. J. Jung ${ }^{2}$, Y. M. Kuo $0^{1,3}$. ${ }^{1}$ College of Medicine, National Taiwan University, Taipei, Taiwan, Republic of China; ${ }^{2}$ School of Medicine, College of Medicine, Taipei Medical University, Department of Microbiology and Immunology, Taipei, Taiwan, Republic of China; ${ }^{3}$ College of Medicine, National Taiwan University, Graduate Institute of Clinical Medicine and Dept of Internal Medicine, Taipei, Taiwan, Republic of China

Background: Streptococcal infection has well known to cause rheumatic fever with various presentations similar to SLE. Besides, both streptococcal-blood stream infection and SLE had aggravated neutrophil extracellular traps (NETs) formation. Previously, we did report a streptococcal induced endocarditis rat model, and identified layers of neutrophil extracellular traps (NETs). According to our findings, specific immunoglobulin $\mathrm{G}(\mathrm{IgG})$ could bridge bacteria to host and these IgGs are required to induce the formation of NETs. Whether oral commensal bacteria could induce pathogenic antibodies which promote NET formation remained unknown.

Objectives: So we aimed to search for novel autoantibodies in SLE through antibody repertoire screening which recognize whole proteins derived from Streptococcus mutans, and investigated to find cross-reactive antibodies presented in the serum of lupus patients and do the correlation between serum MPO(myeloperoxidase)-DNA, a marker of NETosis.

Methods: The streptoccocal specific protein (SSP) was identified through LC-MS and by a proteomics survey. We then purified the target protein in streptococci with expression vector, and antibody level will be detected quantitatively. We recruited patients with SLE, other systemic autoimmune diseases (AIDs) patients to elucidate the performance of this biomarker. Besides, we pursued the Basic Local Alignment Search Tool (BLAST) and searched for cross-reactive autoantigens.

Results: 79 lupus patients and 95 patients with other systemic autoimmune disease were enrolled. By using cut-off value 1.06(set according to area under the receiver operating characteristic

curve) of anti-SSP o.d. value (174 samples), 27 of 79 (34.2\%) SLE patients have positive results while only $7 / 95(7.4 \%)$ was detected in control group. The specificity and sensitivity of the anti-SSP for diagnosis of SLE was $92.6 \%$ and $34.2 \%$ respectively. According to BLAST and B cell-epitope prediction algorithms, The P32-55 epitopes were identified and we synthesized a highly immunogenic and surface-accessible epitope related protein. We named the protein to be MIP (mitochondrial immuno-dominant protein.) Most of the sera from SLE patients could recognize MIP and anti-MIP correlated with serum MPO-DNA( $r=0.41$, $\mathrm{p}=0.039$ ).

Conclusion: The novel anti-SSP antibody against commensal streptococcal specific protein can differentiate SLE and other AIDs. Further investigation to determinate whether the anti-SSP antibody or the associated immune-complex could induce or aggravate NETs formation is warranted.

References:

[1] Sci Transl Med 2011; 3(73):73ra19-73ra19.

[2] J Biomed Sci. 2014 17;21:23.

[3] Circulation. 2015; 131:571-581

[4] Nat Med. 2016 22(2):146-53

Disclosure of Interests: : Che-Hao Hsu: None declared, Chiau-Jing Jung: None declared, Yu-Min Kuo Speakers bureau: Novartis, Pfizer, Rothe, Janssen, Abbvie, UCB, Chugai, Bristol Myers Squibb, Amgen and Astellas DOI: 10.1136/annrheumdis-2020-eular.5674

\section{THU0234 \\ INFLUENCE OF THE SPLICING MACHINERY DYSREGULATION ON THE PATHOGENESIS OF LUPUS AND THE KIDNEY INVOLVEMENT}

C. Lopez-Pedrera ${ }^{1}$, M. Luque-Tévar ${ }^{1}$, A. Ibañez-Costa ${ }^{2}$, A. M. Patiño-Trives ${ }^{1}$ M. A. Aguirre' ${ }^{1}$, P. S. Laura' ${ }^{1}$, M. D. C. Abalos-Aguilera ${ }^{1}$, I. Arias de la Rosa ${ }^{1}$, P. Seguí Azpilcueta ${ }^{1}$, M. Espinosa ${ }^{1}$, N. Barbarroja Puerto ${ }^{1}$, E. CollantesEstévez ${ }^{1}$, J. P. Castaño Fuentes ${ }^{2}$, R. M. Luque Huertas ${ }^{2}$, C. Perez-Sanchez ${ }^{1}$. ${ }^{1}$ IMIBIC/Reina Sofia Hospital/University of Cordoba, Córdoba, Spain; ${ }^{2}$ Department of Cell Biology, Physiology and Immunology, University of Cordoba, Córdoba, Spain

\section{Background:}

Objectives: The aim of this study was to evaluate whether alterations in the splicing machinery of immune cells from Systemic Lupus Erythematosus (SLE) patients could influence the development and activity of the disease and the kidney involvement.
Methods: The study was conducted in 41 SLE patients and 34 healthy donors (HD). Monocytes, lymphocytes and neutrophils were purified by immunomagnetic selection. Then, selected elements of the splicing machinery and a set of genes related to inflammation, renal and cardiovascular disease (including interleukins, adipocytokines, chemokines, and oxidative stress markers, among others) were evaluated using a microfluidic qPCR array (Fluidigm). Besides, the inflammatory profile in plasma, including the analysis of 27 proteins, was evaluated by using the Bioplex assay. In parallel, an extensive clinical/serological evaluation was performed; comprising disease activity and cardiovascular and renal involvement along with autoantibodies, complement factors, and acute phase reactants. Correlation and association studies and logistic models among those clinical and analytical parameters were developed. Mechanistic in vitro studies were carried out by incubation of HD-leukocytes with anti-dsDNA-lgG purified from SLE patients and changes promoted in both, splicing machinery and leukocyte inflammatory profile, were assessed.

Results: A significantly altered expression of spliceosome components was found in all the leukocyte subsets: 27,12 and 11 components were differentially expressed in monocytes, lymphocytes and neutrophils, respectively, in SLE patient's vs HD. In parallel, a number of genes codifying for proteins involved in inflammation, fatty acid metabolism, oxidative stress and migration were found altered and correlations with spliceosome components were further identified Besides, those statistical analyses demonstrated multiple links among altered spliceosome components and the clinical profile of these patients, such as the activity of the disease (SLEDAI), the occurrence of obstetric complications and the presence of arterial hypertension. Logistic regression and ROC curve analyses identified a signature composed in each leukocyte subset by 3 altered spliceosome components that could differentiate between SLE and HDs. Remarkably, the levels of a high number of those altered components were associated to the presence of lupus nephritis (LN). Moreover, ROC curve analyses allowed to identify several cell-specific spliceosome components as potential biomarkers of renal disease. In patients with LN we could also identify a distinctive inflammatory profile in plasma in relation to patients without renal involvement, which further correlated with the altered expression of a number of spliceosome components in each leukocyte subset. Lastly, the in vitro treatment of HD leukocytes with anti-dsDNA promoted the alteration of several spliceosome components found also altered in vivo in SLE lymphocytes, monocytes and neutrophils.

Conclusion: 1)The splicing machinery is greatly altered in leukocytes from SLE patients, regulated, at least partially, by anti-dsDNA antibodies and closely related to the activity of the disease, including obstetrical complications, hypertension and lupus nephritis. 2)Specific components of the spliceosome in SLE leukocytes subsets might be used as potential biomarkers to typify the disease, particularly kidney involvement.

Acknowledgments : Funded by ISCIII (PI18/00837 and RIER RD16/0012/0015) co-funded with FEDER.

Disclosure of Interests: : Chary Lopez-Pedrera Grant/research support from: ROCHE and Pfizer., María Luque-Tévar: None declared, Alejandro IbañezCosta: None declared, Alejandra M. Patiño-Trives: None declared, Maria A Aguirre: None declared, Pérez Sánchez Laura: None declared, Maria del Carmen Abalos-Aguilera: None declared, Iván Arias de la Rosa: None declared, Pedro Seguí Azpilcueta: None declared, Mario Espinosa: None declared, Nuria Barbarroja Puerto Grant/research support from: ROCHE and Pfizer., Speakers bureau: ROCHE and Celgene., Eduardo Collantes-Estévez Grant/research support from ROCHE and Pfizer., Speakers bureau: ROCHE, Lilly, Bristol and Celgene., Justo Pastor Castaño Fuentes: None declared, Raúl Miquel Luque Huertas: None declared, Carlos Perez-Sanchez: None declared DOI: 10.1136/annrheumdis-2020-eular.5237

\section{THU0235 \\ VITAMIN D AND INTERFERON SIGNATURE GENE EXPRESSION IN SYSTEMIC LUPUS ERYTHEMATOSUS}

R. Magro ${ }^{1}$, C. Saliba ${ }^{2}$, L. Camilleri ${ }^{2}$, C. Scerri' ${ }^{2}$, A. Borg ${ }^{1}{ }^{1}$ Mater Dei Hospial, Rheumatology, Msida, Malta; ${ }^{2}$ University of Malta, Msida, Malta

Background: Vitamin D deficiency is more prevalent in patients with systemic lupus eythematosus (SLE) as a result of sun avoidance. ${ }^{1}$ The potential negative impact of vitamin D deficiency on SLE disease activity has been shown in a number of studies. ${ }^{2}$ The expression of the interferon signature genes in SLE correlates positively with disease activity, and these genes are thought to mediate the clinical manifestations of the disease. ${ }^{3}$

Objectives: The aim of this study was to establish whether a relationship exists between serum 25-hydroxyvitamin $\mathrm{D}$ level and the interferon signature gene expression in whole blood of SLE patients.

Methods: Informed consent was obtained from 92 SLE patients who were over the age of 18 and who fulfilled the SLICC classification criteria for SLE. The patients were interviewed and blood samples were taken. SLE disease activity was measured by SLE disease activity index-2K (SLEDAI-2K). RNA extraction was performed from whole blood. QuantiGene Plex technology 